\title{
WARM MOLECULAR GAS IN LUMINOUS INFRARED GALAXIES*
}

\author{
N. $\mathrm{Lu}^{1}$, Y. ZhaO ${ }^{1,2,3}$, C. K. Xu ${ }^{1}$, Y. GaO ${ }^{2,3}$, L. Armus ${ }^{4}$, J. M. Mazzarella ${ }^{1}$, K. G. Isaak ${ }^{5}$, A. O. Petric ${ }^{6,7}$, \\ V. Charmandaris ${ }^{8,9,10}$, T. Díaz-Santos ${ }^{4}$, A. S. Evans ${ }^{11,12}$, J. Howell ${ }^{1}$, P. Appleton ${ }^{1}$, H. Inami ${ }^{13}$, K. Iwasawa ${ }^{14}$,

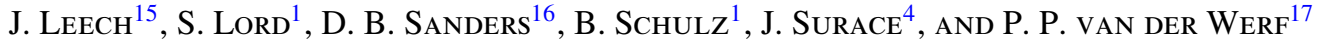 \\ ${ }^{1}$ Infrared Processing and Analysis Center, California Institute of Technology, MS 100-22, Pasadena, CA 91125, USA; lu@ipac.caltech.edu \\ ${ }^{2}$ Purple Mountain Observatory, Chinese Academy of Sciences, Nanjing 210008, China \\ ${ }^{3}$ Key Laboratory of Radio Astronomy, Chinese Academy of Sciences, Nanjing 210008, China \\ ${ }^{4}$ Spitzer Science Center, California Institute of Technology, MS 220-6, Pasadena, CA 91125, USA \\ ${ }^{5}$ ESA Astrophysics Missions Division, ESTEC, P.O. Box 299, 2200-AG Noordwijk, The Netherlands \\ ${ }^{6}$ Gemini Observatory, 670 N. A'ohoku Place, Hilo, HI 96720, USA \\ ${ }^{7}$ Astronomy Department, California Institute of Technology, Pasadena, CA 91125, USA \\ ${ }^{8}$ Department of Physics, University of Crete, GR-71003 Heraklion, Greece \\ ${ }^{9}$ IAASARS, National Observatory of Athens, GR-15236, Penteli, Greece \\ ${ }^{10}$ Chercheur Associé, Observatoire de Paris, F-75014 Paris, France \\ ${ }^{11}$ Department of Astronomy, University of Virginia, 530 McCormick Road, Charlottesville, VA 22904, USA \\ ${ }^{12}$ National Radio Astronomy Observatory, 520 Edgemont Road, Charlottesville, VA 22903, USA \\ ${ }^{13}$ National Optical Astronomy Observatory, Tucson, AZ 85719, USA \\ ${ }^{14}$ ICREA and Institut de Ciències del Cosmos (ICC), Universitat de Barcelona (IEEC-UB), Martí i Franquès 1, E-08028 Barcelona, Spain \\ ${ }^{15}$ Department of Physics, University of Oxford, Denys Wilkinson Building, Keble Road, Oxford OX1 3RH, UK \\ ${ }^{16}$ Institute for Astronomy, University of Hawaii, 2680 Woodlawn Drive, Honolulu, HI 96822, USA \\ ${ }^{17}$ Leiden Observatory, Leiden University, P.O. Box 9513, 2300-RA Leiden, The Netherlands \\ Received 2014 March 25; accepted 2014 May 1; published 2014 May 13
}

\begin{abstract}
We present our initial results on the CO rotational spectral line energy distribution (SLED) of the $J$ to $J-1$ transitions from $J=4$ up to 13 from Herschel SPIRE spectroscopic observations of 65 luminous infrared galaxies (LIRGs) in the Great Observatories All-Sky LIRG Survey. The observed SLEDs change on average from one peaking at $J \leqslant 4$ to a broad distribution peaking around $J \sim 6$ to 7 as the IRAS 60-to-100 $\mu \mathrm{m}$ color, $C(60 / 100)$, increases. However, the ratios of a CO line luminosity to the total infrared luminosity, $L_{\mathrm{IR}}$, show the smallest variation for $J$ around 6 or 7. This suggests that, for most LIRGs, ongoing star formation (SF) is also responsible for a warm gas component that emits CO lines primarily in the mid- $J$ regime $(5 \lesssim J \lesssim 10)$. As a result, the logarithmic ratios of the CO line luminosity summed over CO (5-4), (6-5), (7-6), (8-7) and (10-9) transitions to $L_{\mathrm{IR}}, \log R_{\text {midCO }}$, remain largely independent of $C(60 / 100)$, and show a mean value of $-4.13\left(\equiv \log R_{\text {midCO }}^{\mathrm{SF}}\right.$ ) and a sample standard deviation of only 0.10 for the SF-dominated galaxies. Including additional galaxies from the literature, we show, albeit with a small number of cases, the possibility that galaxies, which bear powerful interstellar shocks unrelated to the current $\mathrm{SF}$, and galaxies, in which an energetic active galactic nucleus contributes significantly to the bolometric luminosity, have their $R_{\text {midCo }}$ higher and lower than $R_{\text {midCO }}^{\mathrm{SF}}$, respectively.
\end{abstract}

Key words: galaxies: active - galaxies: ISM - galaxies: star formation - infrared: galaxies - ISM: molecules submillimeter: galaxies

\section{INTRODUCTION}

Luminous infrared galaxies (LIRGs; defined as $\left.L_{\mathrm{IR}}(8-1000 \mu \mathrm{m}) \geqslant 10^{11} L_{\odot}\right)$ dominate the cosmic star formation (SF) at $z>1$ (Le Flóch et al. 2005; Magnelli et al. 2009). The local counterparts are all known to be rich in molecular gas (Sanders \& Mirabel 1996). While the CO (1-0) line has been widely used to trace the total molecular gas content, SF occurs in the denser parts of molecular gas as evidenced by correlations between $L_{\mathrm{IR}}$ and dense gas tracers, such as HCN (1-0) (e.g., Gao \& Solomon 2004; Wu et al. 2005). SF is expected to heat up the molecular gas substantially. The resulting warm gas can be better traced by mid- $J \mathrm{CO}$ line transitions such as $\mathrm{CO}(6-5)$, which has a critical density of $\sim 3 \times 10^{5} \mathrm{~cm}^{-3}$ and an excitation temperature of $\sim 116 \mathrm{~K}$ (Carilli \& Walter 2013), hinted already by limited ground-based CO data (e.g., Bayet et al. 2009).

We have carried out a survey of warm molecular gas for a sample of 125 LIRGs belonging to the Great Observatories AllSky LIRG Survey (GOALS; Armus et al. 2009), by obtaining

\footnotetext{
* Based on Herschel observations. Herschel is an ESA space observatory with science instruments provided by European-led Principal Investigator consortia and with important participation from NASA.
}

line fluxes of the CO $J$ to $J-1$ transitions from $J=4$ up to 13 with the SPIRE Fourier Transform Spectrometer (FTS; Griffin et al. 2010) on-board Herschel (Pilbratt et al. 2010). The other targeted lines include [N II] $205 \mu \mathrm{m}$, [C I] $609 \mu \mathrm{m}$, and [C I] $370 \mu \mathrm{m}$. The program and observational data on individual galaxies will be given in full in a future paper (N. Lu et al. 2014; in preparation). An earlier Letter (Zhao et al. 2013) presented the initial results on how the [N II] $205 \mu \mathrm{m}$ line correlates with the SF rate. In this Letter we report our initial findings on the observed CO line emission for 65 sample galaxies from the early Herschel observations. We provide a brief description of our survey program and data reduction in Section 2, and present our results and discuss physical implications on gas and dust heating in Section 3.

\section{THE SAMPLE, OBSERVATIONS, AND DATA REDUCTION}

Our full FTS sample is a flux-limited subset of the GOALS sample, which contains 202 LIRGs down to $f_{v}(60 \mu \mathrm{m})=$ $5.24 \mathrm{Jy}$, by further satisfying $F_{\mathrm{IR}}(8-1000 \mu \mathrm{m})>6.5 \times$ $10^{-13} \mathrm{~W} \mathrm{~m}^{-2}$, where $F_{\mathrm{IR}}$ is as defined in Sanders \& Mirabel (1996) and the conversion between $F_{\mathrm{IR}}$ and $L_{\mathrm{IR}}$ used the 


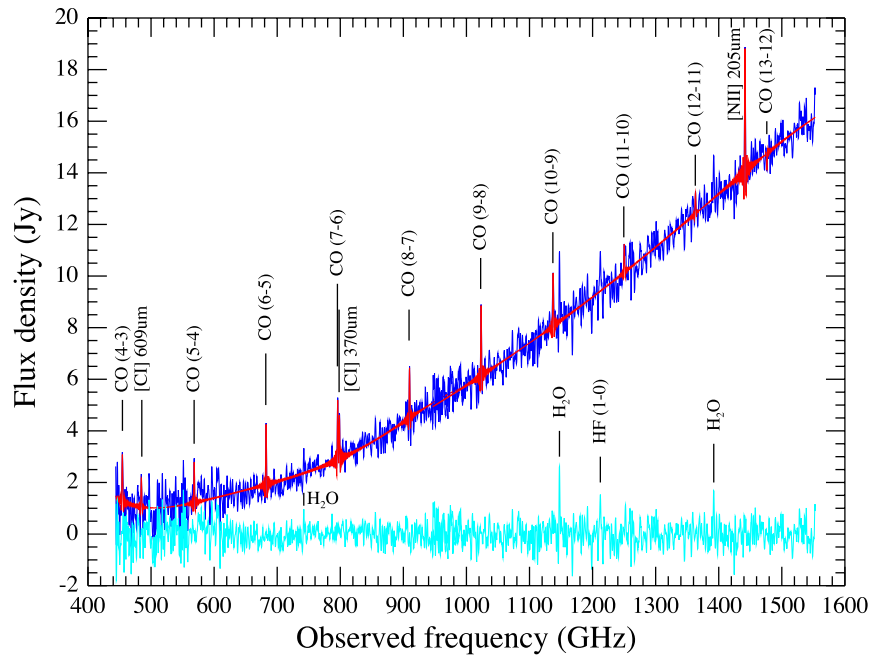

Figure 1. Observed SPIRE spectrum (in blue) of IRAS 17578-0400 (R.A. = $18^{\mathrm{h}} 00^{\mathrm{m}} 31^{\mathrm{s}} .86$, decl. $\left.=-4^{\circ} 00^{\prime} 53^{\prime \prime} .3 ; \mathrm{J} 2000\right)$, a starburst with $\log L_{\mathrm{IR}} / L_{\odot}=$ 11.40. The composite fit of the continuum and the selected lines in $\mathrm{CO},[\mathrm{C} \mathrm{I}]$ and $[\mathrm{N} \mathrm{II}]$ (see the text) is shown in red. Other detected molecular lines were not fit here, but marked in the residual spectrum (in cyan).

luminosity distance given in Table 1 in Armus et al. (2009). Galaxies in a pair were treated separately with their individual $L_{\mathrm{IR}}$ derived as in Díaz-Santos et al. (2010). Our FTS sample contains seven galaxies with $L_{\mathrm{IR}}>10^{12} L_{\odot}$. Ninety-three galaxies were eventually observed by us (program ID: OT1_nlu_1) using the high resolution, sparse mode centered on each galaxy. The remainder have archived FTS data. Our own program emphasized detections of the mid- $J$ CO lines (i.e., $5 \lesssim J \lesssim 10$ ). The on-target integration times range from 1332 to $7992 \mathrm{~s}$, set to detect the anticipated $\mathrm{CO}(6-5)$ flux at $\mathrm{S} / \mathrm{N}>5$.

The 65 galaxies chosen for this Letter are practically pointlike sources with respect to the SPIRE beams (see Zhao et al. 2013), including 38 from own observations, 24 archived observations originally from the HerCULES program (PI: P. van der Werf; van der Werf et al. 2010), UGC 05101 and NGC 7130 from Pereira-Santaella et al. (2013), and Arp 220 from Rangwala et al. (2011). The SPIRE data were reduced homogeneously using Herschel Interactive Processing Environment (HIPE), version 9 (Ott 2010), which offers a line flux accuracy better than $10 \%$. The final spectrum was extracted from the two central detectors (SSWD4 and SLWC3). As an example, Figure 1 shows the spectrum of IRAS 17578-0400, a starburst with $\log L_{\mathrm{IR}} / L_{\odot}=11.40$ and IRAS 60-to- $100 \mu \mathrm{m}$ flux density ratio (also referred to as FIR color), $C(60 / 100) \sim 0.83$, with the fitted $\mathrm{CO},[\mathrm{C} \mathrm{I}]$ ad $[\mathrm{N}$ II] lines marked.

We used the spectrum fitter in HIPE to simultaneously fit a polynomial (of order 5) to the continuum and SINC functions in frequency to the targeted lines. For each SINC profile, only its FWHM was fixed at $1.44 \mathrm{GHz}$. A line was regarded as being detected if (1) the fitted line flux is greater than three times the r.m.s. noise, measured within a frequency interval of 20 FWHM wide, centered on the line frequency in the residual spectrum, and (2) its inferred line velocity difference with the (usually) brightest [N II] $205 \mu \mathrm{m}$ line is within the expected uncertainty based on the spectral resolution and data sampling frequency. The SINC line profiles were adequate for all the CO lines detected in nearly all sources. The only clear exception was NGC 6240, for which the broad CO lines are partially resolved and were fit with a Gaussian convolved profile using the algorithm described in Zhao et al. (2013).

\section{RESULTS AND DISCUSSION}

\subsection{CO Spectral Line Energy Distribution}

Figure 2 shows nine examples of the observed $\mathrm{CO}$ spectral line energy distributions (SLEDs) from our own observations, in order of increasing $L_{\mathrm{IR}}$. These plots illustrate that $L_{\mathrm{IR}}$ is not the best predictor for the SLED shapes. For example, CGCG 448-020 and ESO 069-IG006 are similar in $L_{\mathrm{IR}}$,

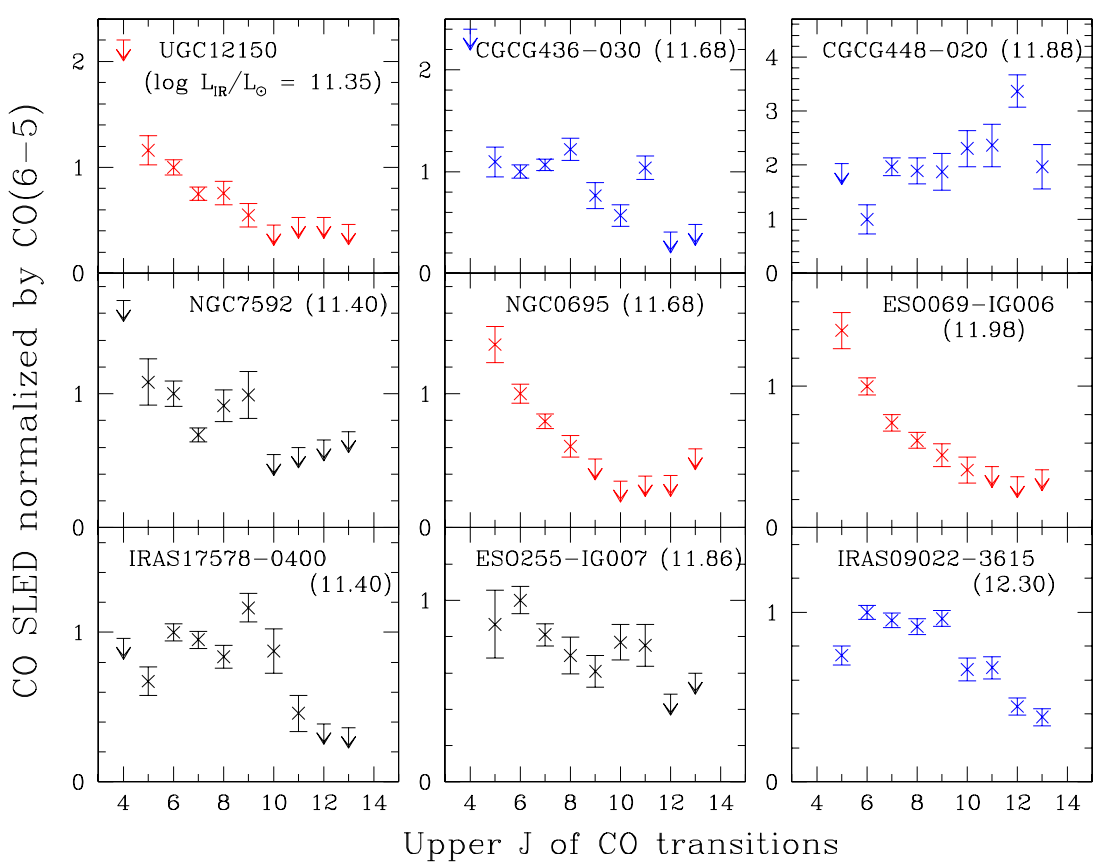

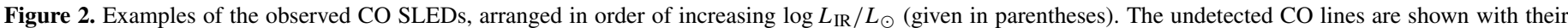

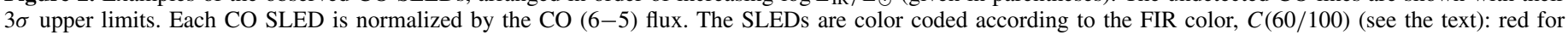

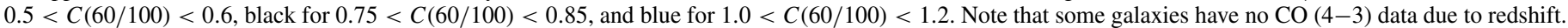




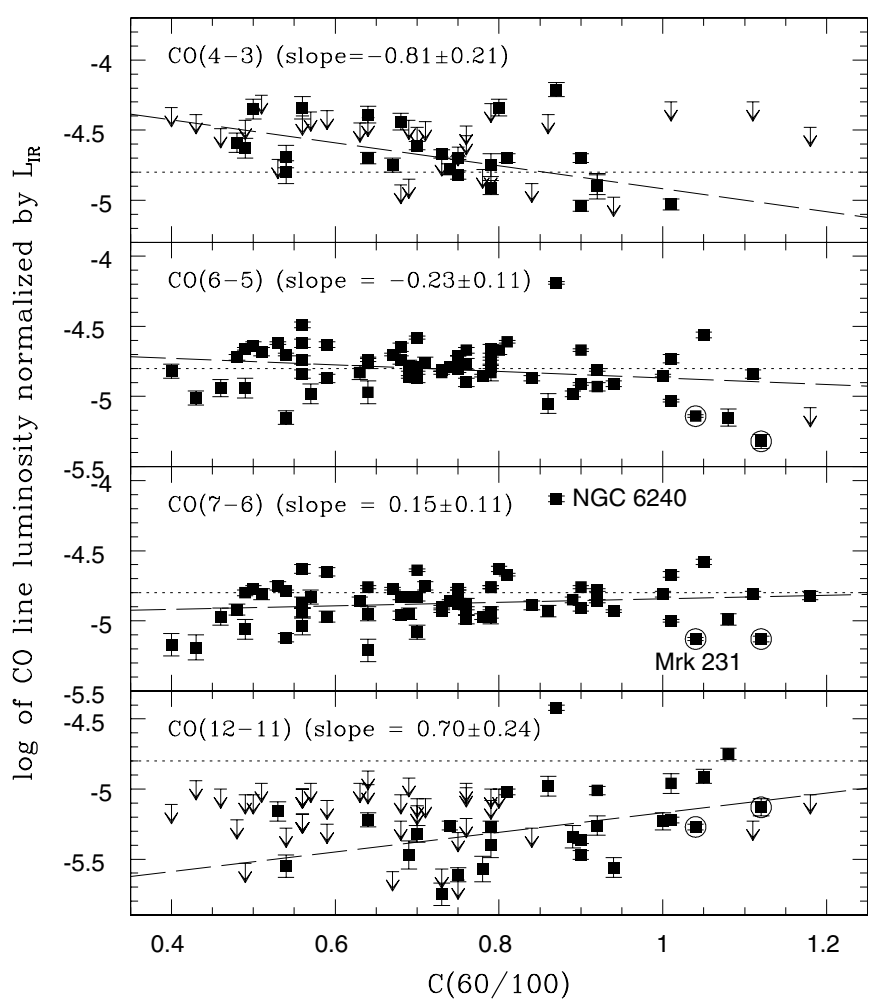

Figure 3. Plots of the logarithmic luminosities of selected $\mathrm{CO}$ lines, each normalized by $L_{\mathrm{IR}}$, as a function the FIR color for our sample. The $\mathrm{CO}$ transition is labeled in each plot. The two galaxies with energetic AGNs (see the text) are enclosed by circles. The arrows indicate the $3 \sigma$ upper limits of an undetected line. In each plot, the dashed line is the least-squares fit to the detections only (but excluding NGC 6240), of which the slope is given. The dotted line in each plot is fixed at -4.8 . Both NGC 6240 and Mrk 231 are marked. Note that some galaxies lack the $\mathrm{CO}(4-3)$ data due to redshift.

but have very different CO SLEDs: the SLED of the former peaks at $J>10$ while that of the latter peaks at $J \lesssim 5$. This difference is mainly due to their different dust temperatures: $C(60 / 100)=1.08$ for CGCG 448-020 versus 0.56 for ESO 069-IG006. To show how $C(60 / 100)$, which measures the average intensity of the dust heating radiation field (Tuffs \& Popescu 2003), correlates with the SLED shape, we color-coded the SLEDs according to $C(60 / 100)$ in Figure 2. Almost all the red-colored SLEDs, with $C(60 / 100) \leqslant 0.6$, peak at $J \lesssim 5$ with the line fluxes decreasing rapidly as $J$ increases. In contrast, the blue-colored SLEDs, with $C(60 / 100)>1.0$, are dominated by a broad distribution over $5 \lesssim J \lesssim 10$. This suggests that it is the intensity of the radiation field, rather than $L_{\mathrm{IR}}$, which determines the SLED shape.

Figure 3 plots the logarithmic $\mathrm{CO}$ line luminosity, normalized by $L_{\mathrm{IR}}$, as a function of $C(60 / 100)$ for $\mathrm{CO}(4-3), \mathrm{CO}(6-5)$, $\mathrm{CO}(7-6)$ and $\mathrm{CO}(12-11)$. An undetected $\mathrm{CO}$ line is shown with its $3 \sigma$ upper limit. The average fractional active galactic nucleus (AGN) contribution to the bolometric luminosity, $f_{\mathrm{AGN}}$, has been derived for most of the GOALS galaxies. This was from a set of the mid-IR diagnostics based on [Ne v]/[ $\mathrm{Ne}$ II], [O IV]/[Ne II], continuum slope, polycyclic aromatic hydrocarbon equivalent width, and the diagram of Laurent et al. (2000), following the prescriptions in Armus et al. (2007; see also Petric et al. 2011; Stierwalt et al. 2013). Of the galaxies used here, only two have $f_{\mathrm{AGN}}>40 \%$ (i.e., $56 \%$ for Mrk 231 and $60 \%$ for IRASF $05189-2524$ ). The remainder all have $f_{\text {AGN }}<40 \%$, including only three galaxies with $f_{\text {AGN }}$ between
$30 \%$ and $40 \%$, and are thus SF dominated. In Figure 3, the two galaxies with $f_{\mathrm{AGN}}>40 \%$ are further enclosed by a circle.

All the plots in Figure 3 span 1.6 dex vertically so that the sample dispersions can be visually compared. The dotted line in each plot helps in identifying the most energetic CO line at any given $C(60 / 100)$ by noting that a galaxy lies on a vertical line across the plots. Figure 3 shows that as $C(60 / 100)$ increases, the overall $\mathrm{CO}$ gas gets warmer: The slope of the linear fit (i.e., the dashed line in each plot) to the detections only (but excluding the outlier NGC 6240) increases monotonically from about -0.8 for $\mathrm{CO}(4-3)$ to +0.7 for $\mathrm{CO}(12-11)$. Both plots of $\mathrm{CO}(4-3)$ and $\mathrm{CO}(12-11)$ contain quite a few upper limits. We performed two tests on whether the fitted slope could be substantially biased due to the non-detections: (1) a full regression including the upper limits based on a survival analysis outlined by Isobe et al. (1986), and (2) using only the detections from the 27 galaxies with the more sensitive $\mathrm{CO}$ data from the Herschel archive, for which there are fewer upper limits. The results from both tests confirm, within the fit uncertainties, that the slopes derived from the fits to the detections are representative of the entire sample.

A more significant message from Figure 3 is that the ratio of $\mathrm{CO}(6-5)$ or $\mathrm{CO}(7-6)$ to the IR emission is rather constant over the range of $C(60 / 100)$ probed. This argues for at least two gas components: a warm component, which emits the $\mathrm{CO}$ lines primarily in mid- $J$ (i.e., $5 \lesssim J \lesssim 10$ ) and correlates best with the dust emission, is mainly responsible for the observed constant ratio seen around $J=6$ or 7 . Since the dominant heating source for the IR emission is current SF, the same ongoing SF should be also responsible for this warm $\mathrm{CO}$ gas component. The other component is a "cold" and predominantly less dense gas that emits CO lines primarily at $J \lesssim 4$ and is not directly related to current SF. As $C(60 / 100)$ increases, the warm gas component becomes more energetic, resulting in the trend seen in Figures 2 and 3. As we argue in Section 3.2, in galaxies with a prominent AGN, there could also be a meaningful third component of hot and likely denser gas, which emits CO lines primarily at $J \gtrsim 10$ and is thus not sampled adequately by the SPIRE spectrometer.

\subsection{Molecular Gas and Dust Heating}

Various heating mechanisms have been considered in the literature for warm $\mathrm{CO}$ line emission in galaxies, including far-UV photon heating from massive stars (hereafter referred to as photon-dominated region or PDR scenario; e.g., IC 342, Rigopoulou et al. 2013), X-ray photon heating from AGNs (referred to as X-ray dominated region or XDR scenario; e.g., Mrk 231, van der Werf et al. 2010; NGC 1068, Spinoglio et al. 2012), heating by cosmic-rays enhanced from supernovae (e.g., NGC 253; Bradford et al. 2003), and interstellar shocks (e.g., Flower \& Pineau Des Forêts 2010). The shock scenario has been favored in many cases, including M82 (Kamenetzky et al. 2012), Arp 220 (Rangwala et al. 2011), NGC 891 (Nikola et al. 2011), NGC 253 (Rosenberg et al. 2014), NGC 6240 (Meijerink et al. 2013), and NGC 1266 (Pellegrini et al. 2013). In this Letter, we further divide the shock scenario into two categories: (A) shocks associated with processes derived from current SF, such as supernovae and stellar winds, and (B) shocks that derive from energy sources other than current SF, such as those associated with AGN-driven gas outflows, radio jets, or galaxy-galaxy collision. While studies of XDR point to CO SLEDs peaking well beyond mid-J (e.g., Spaans \& Meijerink 2008), all the other heating mechanisms can, in principle, produce similar CO 


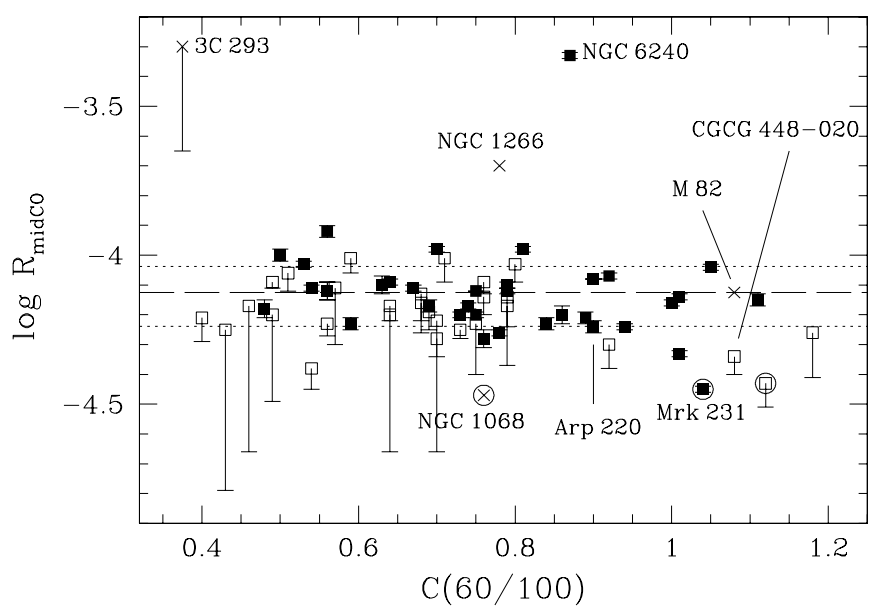

Figure 4. Plot of $\log$ of $R_{\text {midCO }}$, the ratio of the combined luminosity from the representative mid- $J$ CO lines of $\mathrm{CO}(5-4),(6-5),(7-6),(8-7)$ and $(10-9)$ to $L_{\mathrm{IR}}$, as a function of the FIR color for our sample galaxies (solid or open squares) and four additional galaxies from the literature (crosses). The galaxies with a significant AGN are further enclosed by a circle. For those with one or two CO lines below the $3 \sigma$ detection threshold, they are shown by an open square or cross that represents the ratio when the $3 \sigma$ upper limits are used for the undetected lines, with a one-sided line extending to the ratio as though the undetected lines had zero fluxes. The sample average and standard deviation for the SF-dominated galaxies in our own sample (excluding NGC 6240) are marked by the dashed and dotted lines, respectively (see the text). A few galaxies are labeled here and discussed in the text.

SLEDs within the mid- $J$ regime, making them difficult to be differentiated based on SPIRE CO data alone.

In Figure 4, we sum over the mid- $J$ CO lines of $J=5$, $6,7,8$, and 10 , and plot in $\log$ the resulting luminosity to $L_{\mathrm{IR}}$ ratio, $R_{\text {midCo, }}$ as a function of $C(60 / 100)$. [The CO $(9-8)$ line was left out because the FTS spectra are usually noisier around its frequency.] Our sample galaxies are in solid or open squares, with the latter representing those with one or more of the CO lines undetected. For these galaxies, we present the possible range for $R_{\text {midCo }}$ between the 0 and $3 \sigma$ values for the undetected lines. In most of these cases, the actual ratio should be much closer to the upper end of the range as most of the undetected lines in the mid- $J$ regime have a fitted line flux not far below the $3 \sigma$ threshold. Overall, $R_{\text {midCo }}$ shows little systematic dependence on $C(60 / 100)$, except for (1) $C(60 / 100) \gtrsim 1$, where more data points (e.g., Mrk 231) tend to lie below the sample mean, and (2) the clear outlier NGC 6240. As comparison cases, we also plot in Figure 4 four additional galaxies indicated by crosses. Three are from the references listed above: M82, an archetypical starburst; NGC 1266, a disk galaxy with possible AGN-driven molecular outflows (Alatalo et al. 2011); and NGC 1068, a well-known Seyfert galaxy with its $\mathrm{CO}$ line emission detected up to $J=$ 30 (Hailey-Dunsheath et al. 2012) and $f_{\mathrm{AGN}} \sim 50 \%$ (Telesco \& Decher 1988). M82 is slightly extended and its $L_{\mathrm{IR}}$ used here was limited to within the same beam size as for the CO SLED. The fourth object is $3 \mathrm{C} 293$, a radio galaxy rich in warm $\mathrm{H}_{2}$, possibly excited by radio jet-driven shocks (Ogle et al. 2010). From the archival SPIRE spectrum of 3C 293 (Obs. ID: 1342238242; PI: P. Papadopoulos), we derived the fluxes of 1.69, 2.91 and $2.14 \times 10^{-18} \mathrm{~W} \mathrm{~m}^{-2}$ for $\mathrm{CO}(6-5), \mathrm{CO}(7-6)$ and $\mathrm{CO}(8-7)$, respectively, all at $\mathrm{S} / \mathrm{N} \gtrsim 3$, but only the $3 \sigma$ upper limits of 5.49 and $3.15 \times 10^{-18} \mathrm{~W} \mathrm{~m}^{-2}$ for $\mathrm{CO}(5-4)$ and $\mathrm{CO}(10-9)$, respectively. Its $F_{\mathrm{IR}} \approx 3.07 \times 10^{-14} \mathrm{~W} \mathrm{~m}^{-2}$, based on an $12 \mu \mathrm{m}$ flux from Siebenmorgen et al. (2004) and IRAS flux densities at 25, 60 and $100 \mu \mathrm{m}$ from Golombek et al. (1988). The galaxy
3C 293 is shown in Figure 4 by its possible range in $R_{\text {midCo. In }}$ both NGC 1266 and 3C 293, our estimated $f_{\text {AGN }}<10 \%$, based on [O IV]/[Ne II] from Dudik et al. (2009) or Ogle et al. (2010).

The relatively small sample scatter in Figure 4 for the SFdominated LIRGs (except for NGC 6240) suggests convincingly that the current SF is also the main heating source for the mid$J$ CO line emission. If we exclude NGC 6240 on one side of the main locus and the two AGNs from our sample on the other side, the average and sample standard deviation of $\log R_{\text {midCO }}$ from the remaining detections in our own sample are -4.13 ( $\equiv \log R_{\text {midCo }}^{\mathrm{SF}}$; i.e., the dashed line in Figure 4) and 0.10 ( $\equiv \log \sigma_{\mathrm{SF}}$; marked by the two dotted lines in Figure 4 ), respectively.

NGC 6240 has $R_{\text {midCo }} / R_{\text {midCO }}^{\mathrm{SF}} \sim 6.3$, which is $\sim 8 \log \sigma_{\mathrm{SF}}$

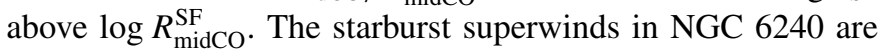
believed to power the large-scale diffuse ionized gas (e.g., Heckman et al. 1987) and shock-excited $\mathrm{H}_{2}$ line emission (e.g., Max et al. 2005). It is not clear how a category-A shock case can lead to such a high $R_{\text {midCo. The energy output of stellar winds }}$ should scale in certain way with that of the far-UV photons powering $L_{\mathrm{IR}}$, resulting in $R_{\text {midCO }}$ around some characteristic value. Indeed, all the other superwind galaxies (e.g., Arp 220 and M82) show a "normal" $R_{\text {midCo. }}$ The fact that no galaxies are seen at intermediate $R_{\text {midCO }}$ values argues against the hypothesis that NGC 6240 represents an optimal wind-gas coupling efficiency. Therefore, we can not rule out the possibility that some forms of category-B shocks might also be at work in the nuclear region of NGC 6240, where the gas is highly turbulent (Tacconi et al. 1999) and the heating source of the warm $\mathrm{H}_{2}$ emission is still controversial, with arguments for (e.g., Ohyama et al. 2000) and against (e.g., Feruglio et al. 2013) a superwind case. A category$\mathrm{B}$ shock scenario can in principle explain a higher $R_{\text {midCO }}$ as there is little far-UV counterpart. Indeed, both NGC 1266 and 3C 293, where the warm $\mathrm{CO}$ emission might be enhanced respectively by the AGN- and radio jet-driven shocks, show a higher $R_{\text {midCO }}$ in Figure 4.

On the other hand, both Mrk 231 and NGC 1068 have a lower $R_{\text {midCo }}$ in Figure 4 . They both have significant hot CO gas emitting at $J>10$ (González-Alfonso et al. 2014; HaileyDunsheath et al. 2012), likely associated with XDR (van der Werf et al. 2010; Spinoglio et al. 2012). Furthermore, the model prediction that the XDR-associated CO emission occurs mainly at higher $J$ levels is also supported by the observation that, with comparable $C(60 / 100)$, M82 and Mrk 231 have almost identical CO SLEDs for $J \lesssim 10$ (Pereira-Santaella et al. 2013). These results suggest that an $R_{\text {midCO }}$ significantly lower than $R_{\text {midCO }}^{\mathrm{SF}}$ may be a result of missing most of the XDR-associated CO line cooling at $J>10$. However, not all the galaxies with a lower $\mathrm{CO} / \mathrm{IR}$ ratio in Figure 4 have a powerful AGN. One marked example is CGCG 448-020, of which $L_{\mathrm{IR}}$ is dominated by an extremely compact, extranuclear starburst (Inami et al. 2010). This might hint that a lower $R_{\text {midCo }}$ could also be associated with some rare, extreme starbursts as a large-scale version of those Galactic extreme compact molecular cores where the $\mathrm{CO}$ line emission peaks at $J>10$ (Etxaluze et al. 2013; Habart et al. 2010).

Under the assumption that AGN/XDR results in $\mathrm{CO}$ line emissions mainly at $J>10$, one can use Figure 4 to estimate the fractional AGN contribution to $L_{\mathrm{IR}}$ in an AGN where no significant Category-B shock is present: The measured $\log R_{\text {midCO }} \approx \log R_{\mathrm{midCO}}^{\mathrm{SF}}+\log \left[1-\left(L_{\mathrm{IR}}^{\mathrm{AGN}} / L_{\mathrm{IR}}\right)\right]$, where $L_{\mathrm{IR}}^{\mathrm{AGN}}$ is the AGN component of $L_{\mathrm{IR}}$. For example, the observed $\log R_{\text {midCO }}$ of -4.45 for Mrk 231 implies that $L_{\mathrm{IR}}^{\mathrm{AGN}} / L_{\mathrm{IR}} \sim 52 \%$ 
$( \pm 10 \%)$, in good agreement with $f_{\mathrm{AGN}} \sim 56 \%$ from our mid-IR diagnostics. In the case of NGC 1068 , we have $L_{\mathrm{IR}}^{\mathrm{AGN}} / L_{\mathrm{IR}} \sim$ $54 \%( \pm 10 \%)$, in good agreement with an independent estimate in Telesco \& Decher (1988).

In summary, (1) we demonstrated that the SF-dominated LIRGs show a relatively tight distribution in terms of $\log R_{\text {midCO }}$, with a mean of -4.13 and a sample standard deviation of 0.10 ; and (2) we showed, albeit with a small number of cases, the possibility that (a) galaxies bearing powerful interstellar shocks not associated with current SF and (b) galaxies with a significant AGN contribution to their bolometric luminosity, have their $\log R_{\text {midCo }}$ higher and lower than -4.13 , respectively.

While it is rather conclusive that the heating source of the mid- $J$ CO line emission in most LIRGs is current SF, more work needs to be done to clarify which SF-related mechanisms are directly responsible for the warm CO line emission.

This Letter benefited from a number of useful comments made by an anonymous referee. This work is based in part on observations made with Herschel, a European Space Agency Cornerstone Mission with significant participation by NASA. Support for this work was provided in part by NASA through an award issued by JPL/Caltech. This research has made use of the NASA/IPAC Extragalactic Database (NED), which is operated by Jet Propulsion Laboratory, California Institute of Technology, under contract with NASA.

\section{REFERENCES}

Alatalo, K., Blitz, L., Young, L. M., et al. 2011, ApJ, 735, 88

Armus, L., Charmandaris, V., Bernard-Salas, J., et al. 2007, ApJ, 656, 148

Armus, L., Mazzarella, J. M., Evans, A. S., et al. 2009, PASP, 121, 559

Bayet, E., Gerin, M., Philips, T. G., \& Contursi, A. 2009, MNRAS, 399, 264

Bradford, C. M., Nikola, T., Stacey, G. J., et al. 2003, ApJ, 586, 891

Carilli, C. L., \& Walter, F. 2013, ARA\&A, 51, 105

Díaz-Santos, T., Charmandaris, V., Armus, L., et al. 2010, ApJ, 723, 993

Dudik, R. P., Satyapal, S., \& Marcu, D. 2009, ApJ, 691, 1501

Etxaluze, M., Goicoechea, J. R., Cernicharo, J., et al. 2013, A\&A, 556, A137

Feruglio, C., Fiore, F., Piconcelli, E., et al. 2013, A\&A, 558, A87
Flower, D. R., \& Pineau Des Forêts, G. 2010, MNRAS, 406, 1745

Gao, Y., \& Solomon, P. M. 2004, ApJ, 606, 271

Golombek, D., Miley, G. K., \& Neugebauger, G. 1988, AJ, 95, 26

González-Alfonso, E., Fischer, J., Graciá-Carpio, J., et al. 2014, A\&A, 561, A27

Griffin, M. J., Abergel, A., Abreu, A., et al. 2010, A\&A, 518, L3

Habart, E., Dartois, E., Abergel, A., et al. 2010, A\&A, 518, L116

Hailey-Dunsheath, S., Sturm, E., Fischer, J., et al. 2012, ApJ, 755, 57

Heckman, T. M., Armus, L., \& Miley, G. K. 1987, AJ, 93, 276

Inami, H., Armus, L., Surace, J. A., et al. 2010, AJ, 140, 63

Isobe, T., Feigelson, E. D., \& Nelson, P. I. 1986, ApJ, 306, 490

Kamenetzky, J., Glenn, J., Rangwala, N., et al. 2012, ApJ, 753, 70

Laurent, O., Mirabel, I. F., Charmandaris, V., Gallais, P., et al. 2000, A\&A, 359,887

Le Flóch, E., Papovich, C., Dole, H., et al. 2005, ApJ, 632, 169

Magnelli, B., Elbaz, D., Chary, R. R., et al. 2009, A\&A, 496, 57

Max, C. E., Canalizo, G., Macintosh, B. A., et al. 2005, ApJ, 621, 738

Meijerink, R., Kristensen, L. E., Weiß, A., et al. 2013, ApJL, 762, L16

Nikola, T., Stacey, G. J., Brisbin, D., et al. 2011, ApJ, 742, 88

Ogle, P., Boulanger, F., Guillard, P., et al. 2010, ApJ, 1193

Ohyama, Y., Yoshida, M., Takata, T., et al. 2000, PASJ, 52, 563

Ott, S. 2010, in ASP Conf Ser. 434, Astronomical Data Analysis Software and Systems XIX, ed. Y. Mizumoto, K.-I. Morita, \& M. Ohishi (San Francisco, CA: ASP), 139

Pellegrini, E. W., Smith, J. D., Wolfire, M. G., et al. 2013, ApJL, 779, L19

Pereira-Santaella, M., Spinoglio, L., Busquet, G., et al. 2013, ApJ, 768, 55

Petric, A. O., Armus, L., Howell, J., et al. 2011, ApJ, 730, 28

Pilbratt, G. L., Riedinger, J. R., Passvogel, T., et al. 2010, A\&A, 518, L1

Rangwala, N., Maloney, P. R., Glenn, J., et al. 2011, ApJ, 743, 94

Rigopoulou, D., Hurley, P. D., Swinyard, B. M., et al. 2013, MNRAS, 434, 2051

Rosenberg, M. J. F., Kazandjian, M. V., van der Werf, P. P., et al. 2014, A\&A, 564, A126

Sanders, D. B., \& Mirabel, I. F. 1996, ARA\&A, 34, 749

Siebenmorgen, R., Freudling, W., Krugel, E., \& Baas, M. 2004, A\&A, 421, 129

Spaans, M., \& Meijerink, R. 2008, ApJL, 678, L5

Spinoglio, L., Pereira-Santaella, M., Busquet, G., et al. 2012, ApJ, 758, 108

Stierwalt, S., Armus, L., Surace, J. A., et al. 2013, ApJS, 206, 1

Tacconi, L. J., Genzel, R., Tecza, M., et al. 1999, ApJ, 524, 732

Telesco, C. M., \& Decher, R. 1988, ApJ, 334, 573

Tuffs, R. J., \& Popescu, C. C. 2003, in Exploiting the ISO Data Archive. Infrared Astronomy in the Internet Age, ed. C. Gry et al. (ESA SP-511; Noordwijk: ESA), 239

van der Werf, P. P., Isaak, K. G., Meijerink, R., et al. 2010, A\&A, 518, L42

Wu, J., Evans, N. J., II, Gao, Y., et al. 2005, ApJ, 635, 173

Zhao, Y., Lu, N., Xu, C. K., et al. 2013, ApJL, 765, L13 